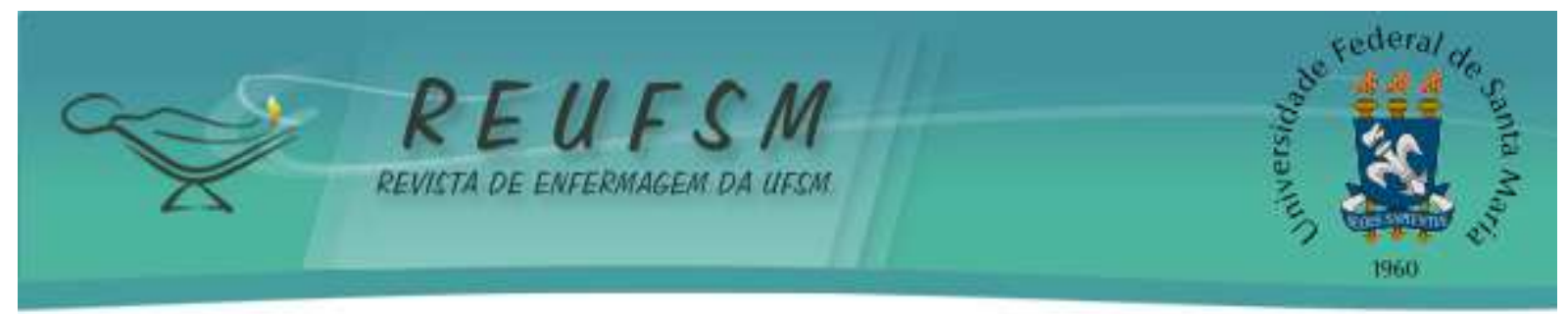

\title{
QUALIDADE DE VIDA DE BOMBEIROS MILITARES ATUANTES NOS SERVIÇOS OPERACIONAL E ADMINISTRATIVO
}

\section{QUALITY OF LIFE OF MILITARY FIREFIGHTERS WHO WORK IN OPERATIONAL AND ADMINISTRATIVE SERVICES}

\section{CALIDAD DE VIDA DE LOS BOMBEROS MILITARES ACTUANTES EN LOS SERVICIOS OPERACIONAL Y ADMINISTRATIVO}

Doi: $10.5902 / 2179769230564$

\author{
Lauane Nogueira dos Santos ${ }^{1}$ \\ Tania Maria Ascari ${ }^{2}$ \\ Clodoaldo Antônio de Sá ${ }^{3}$ \\ Rosana Amora Ascari ${ }^{4}$
}

RESUMO: Objetivo: analisar a qualidade de vida de bombeiros militares atuantes em serviços operacional e administrativo. Método: estudo transversal, de abordagem quantitativa, realizado em um município do oeste catarinense entre março e abril de 2016. Para a coleta de dados utilizou-se o questionário de avaliação da qualidade de vida da Organização Mundial da Saúde, versão curta (WHOQOL-Bref). Utilizou-se o programa Statistical Pack age of Social Sciences para as análises estatísticas. Resultados: os resultados evidenciam que os 51 participantes apresentaram níveis regulares de qualidade de vida, os bombeiros que atuam no operacional apresentam os piores resultados sobre a percepção da qualidade de vida em comparação aos trabalhadores do administrativo $(\mathrm{p}=<0,05)$. A realização de horas extras interferiu negativamente na qualidade de vida $(\mathrm{p}=<0,05)$. No domínio Meio Ambiente foram encontrados os piores valores. Conclusão: os participantes avaliaram sua qualidade de vida como regular o que pode repercutir na saúde e bem-estar, assim como interferir no seu cotidiano de trabalho. Sugere-se a utilização deste indicador na gestão da saúde dos trabalhadores.

Descritores: Saúde do trabalhador; Qualidade de vida; Esgotamento profissional; Bombeiro

ABSTRACT: Aim: to analyze the quality of life of military firefighters active in operational and administrative services. Method: a cross - sectional study with a quantitative approach, carried out in a municipality in the west of Santa Catarina from March to April 2016.The World Health Organization quality of life assessment questionnaire (WHOQOL-Bref) was used to collect data. Results: the results show that the 51 participants had regular levels of quality of life, firefighters operating at the operational level had the worst results on the perception of quality of life compared to the administrative workers $(p=<0.05)$. Overtime workload interfered negatively in quality of life $(p=<0.05)$. In the Environment domain the worst values were found. Conclusion: the participants assessed their quality of life as

\footnotetext{
1 Enfermeira. Universidade do Estado de Santa Catarina (UDESC). Chapecó (SC), Brasil. E-mail: lauane.nogueira@gmail.com

2 Enfermeira e Psicóloga. Mestre em Enfermagem. Professora da Universidade do Estado de Santa Catarina/UDESC. Chapecó (SC), Brasil. E-mail: tania.ascari@udesc.br

${ }^{3}$ Educador Físico. Doutor em Ciência do Movimento Humano. Professor da Universidade Comunitária da Região de Chapecó (UNOCHAPECÓ). Coordenador do Programa de Pós-Graduação Stricto Sensu em Ciências da Saúde da Unochapecó. Chapecó (SC), Brasil. E-mail: clodoaldo@unochapeco.edu.br

${ }^{4}$ Enfermeira. Doutora em Enfermagem. Professora da Universidade do Estado de Santa Catarina/UDESC. Chapecó (SC), Brasil. E-mail: rosana.ascari@ hotmail.com
} 


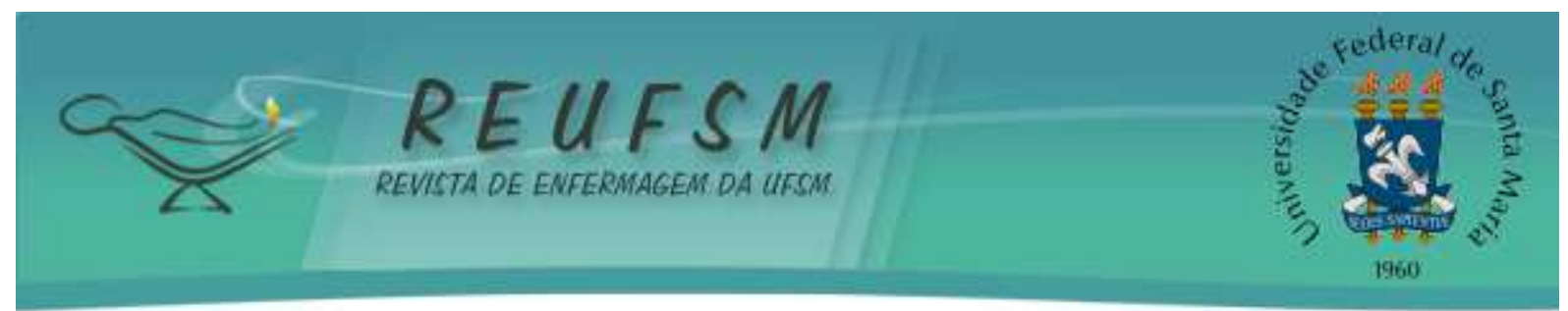

regular, which may affect health and well-being, as well as interfering in their daily work. The use of this indicator is, hence, suggested, in the health management of workers.

Descriptors: Occupational health; Quality of life; Burnout, professional; Firefighter

RESUMEN: Objetivo: analizar la calidad de vida de los bomberos militares actuantes en servicios operativos y administrativos. Método: estudio transversal, de carácter cuantitativo, realizado en un municipio del oeste de Santa Catarina entre marzo y abril de 2016. Para la recolección de datos se utilizó el cuestionario de evaluación de la calidad de vida de la Organización Mundial de la Salud, versión corta (WHOQOL-Bref). Se utilizó el programa Statistical Package of Social Sciences para los análisis estadísticos. Resultados: los resultados evidenciaron que los 51 participantes presentaron niveles regulares de calidad de vida, los bomberos que actúan en el operativo presentan los peores resultados sobre el entendimiento de su calidad de vida en comparación con los trabajadores del administrativo $(p=<0,05)$. La realización de horas extras influyó negativamente en la calidad de vida ( $p=$ <0,05). En el campo Medio Ambiente fue identificado los peores valores. Conclusión: los participantes evaluaron su calidad de vida como regular, lo que puede resultar en la salud y en el bienestar, así como interferir en el cotidiano de trabajo. Se sugiere la utilización de ese indicador en la gestión de la salud de los trabajadores.

Descriptores: Salud laboral; Calidad de vida; Agotamiento profesional; Bombero

\section{INTRODUÇÃO}

A profissão bombeiro é uma atividade que exige conhecimento, preparo físico e psicológico para enfrentar situações desgastantes. O convívio diário com o perigo e o sofrimento humano expõe os bombeiros a desafios constantes para lidar com situações de estresse no trabalho. São cobrados por respostas eficientes frentes a situações de emergência gerando desgaste físico e mental. ${ }^{1}$

O Corpo de Bombeiros Militares do Estado de Santa Catarina (SC) é regulamentado pela Constituição Estadual de 2009, caracterizado em seu Art. 108 como:2:66 "órgão permanente, força auxiliar, reserva do Exército, organizado com base na hierarquia e disciplina, subordinado ao Governador do Estado", o qual cabe, "realizar os serviços de prevenção de sinistros ou catástrofes, de combate a incêndio e de busca e salvamento de pessoas e bens e o atendimento pré-hospitalar; [...]". 2:66

Por suas características, a atividade laboral dos bombeiros militares pode trazer consequências para a sua qualidade de vida (QV), que é definida como "a percepção do indivíduo sobre a sua posição na vida, no contexto da cultura e dos sistemas de valores nos quais ele vive, em relação às suas metas, expectativas, critérios e interesses [...]".:1405 


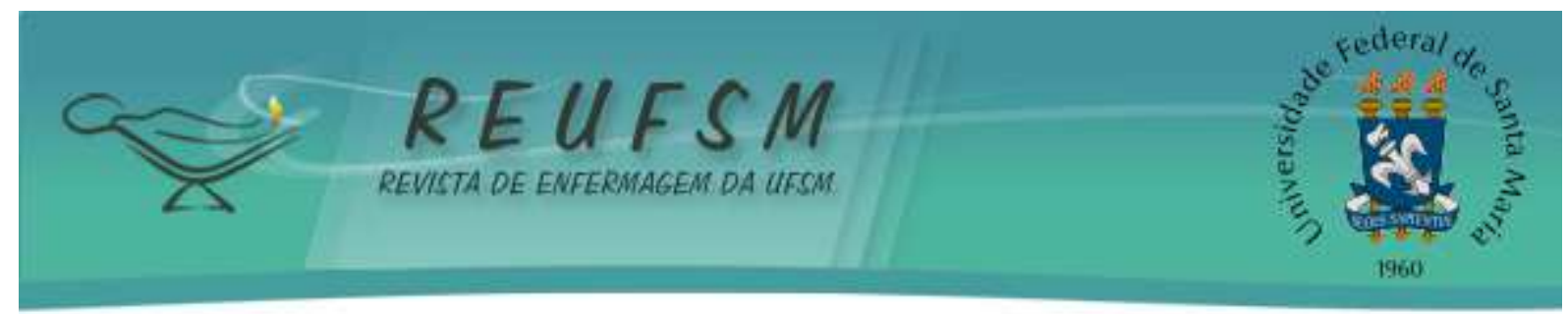

O adoecimento laboral traz prejuízos aos trabalhadores, além de onerar empregadores e a sociedade. As condições de estresse laboral são potencializadas em profissões nas quais os sujeitos lidam cotidianamente com os problemas ou o sofrimento humano, ou em situações nas quais esses profissionais se envolvem intensamente com as dificuldades psicológicas, sociais e físicas das pessoas que fazem parte do seu meio de trabalho, características bastante marcantes da profissão de bombeiro, ${ }^{4}$ e que pode influenciar negativamente a qualidade de vida.

Exigidos de respostas rápidas frentes a situações de emergência e competência de decisão, os bombeiros são expostos ao estresse intenso e incidentes críticos de gravidade variada, situações que provocam um desgaste lento da capacidade física e psicológica, podendo gerar uma diminuição da satisfação do trabalho, negatividade ao profissional e a quem solicita serviços. ${ }^{1}$

Levando em consideração que os trabalhadores podem adoecer ou morrer por causas relacionadas ao trabalho, como consequência da profissão que exercem ou exerceram no passado, ou pelas condições em que seu trabalho é, ou foi realizado, ${ }^{5}$ e a escassez de estudos abordando a temática qualidade de vida em bombeiros, emergiu a seguinte questão de pesquisa: Qual a avaliação de bombeiros militares atuantes em serviços operacional e administrativo sobre a qualidade de vida?

O objetivo deste estudo foi analisar a qualidade de vida de bombeiros militares atuantes em serviços operacional e administrativo.

\section{MÉTODO}

Trata-se de um estudo transversal de abordagem quantitativa, realizado no $6^{\circ}$ Batalhão de Bombeiros Militar de Santa Catarina ( $\left.6^{\circ} \mathrm{BBM}-\mathrm{SC}\right)$, na região oeste do Estado, o qual conta com 73 bombeiros militares elegíveis, sendo que 26 atuam no serviço interno/administrativo e 47 respondem pelo Expediente e Central de Emergência (chamadas pelo número 193) e pelo operacional, atuando no atendimento pré-hospitalar, combate a incêndio, resgate veicular, salvamento aquático, prevenções e outros.

Os profissionais do setor operacional estão divididos em escalas de trabalho 24/48 horas. $\mathrm{O}$ administrativo atua em turno diurno de seis horas diárias, responsabilizando-se pelos serviços internos, tais como, aprovação e liberação de projetos de segurança de residências, comércios e outros. 


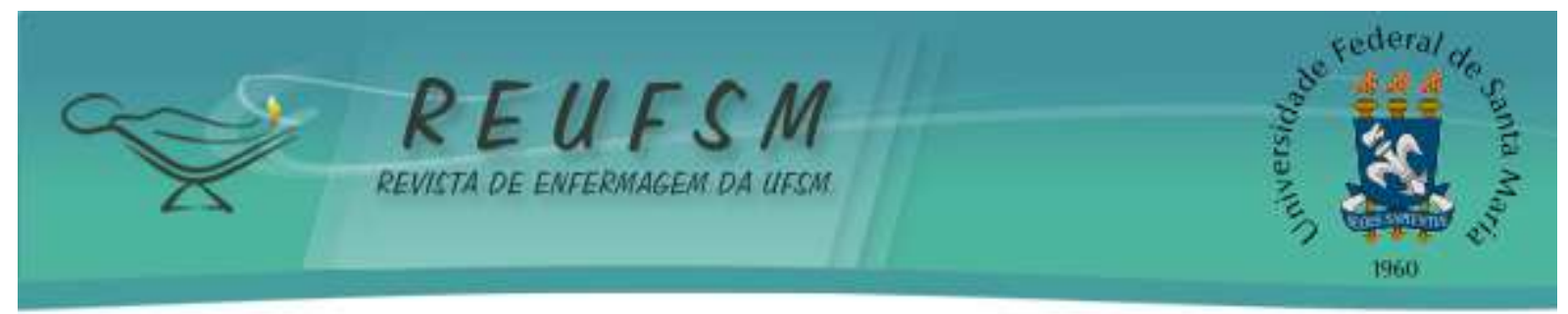

Como critério de inclusão considerou-se todos os bombeiros militares, de ambos os sexos, atuantes $6^{\circ}$ BBM-SC. Foram excluídos do estudo profissionais em operação veraneio, férias, licença, em cursos de formação ou em atestado médico. A coleta de dados ocorreu no período de março e abril de 2016 nas dependências do $6^{\circ} \mathrm{BBM}-\mathrm{SC}$, em diferentes turnos e horários, sendo que cada possível participante foi abordado uma única vez. Para a coleta de dados utilizou-se questionário auto aplicado contendo dados sociodemográficos, informações laborais e dados sobre o estilo de vida, que foi preenchido e devolvido ao pesquisador no mesmo dia. A avaliação da qualidade de vida foi mensurada por meio do Word Health Organization Quality of Life, versão curta (WHOQOL-Bref), adaptado e traduzido no Brasil por pesquisadores do Departamento de Psiquiatria e Medicina Legal da Faculdade de Medicina da Universidade Federal do Rio Grande do Sul (UFRGS), que demonstrou características satisfatórias de consistência interna ( $\alpha$ de Cronbach $=0,91){ }^{6}$

O WHOQOL-Bref aborda quatro domínios da qualidade de vida, o qual procura apresentar inferências específicas quanto à capacidade física, bem-estar psicológico, aos relacionamentos sociais e pessoais e ao meio ambiente, contemplando perguntas com opções de respostas do tipo Likert, com valores de zero a cinco. Propõe também um domínio adicional relacionadas à qualidade de vida global, mediante resposta de duas questões de âmbito mais genérico, sendo uma relacionada à autopercepção da qualidade de vida e satisfação com a saúde. ${ }^{6}$

O escore para cada domínio e avaliação global varia de zero a cem pontos, sendo zero a menor percepção e 100 a maior percepção do indicador de qualidade de vida do domínio em questão. ${ }^{3} \mathrm{O}$ processo de análise de dados obedeceu a seguinte sequência: codificação, tabulação, organização e tratamento estatístico dos dados com auxílio do programa Statistical Pack age for the Social Sciences ${ }^{\circledR}$ (SPSS), versão 22.0 para as análises estatísticas.

A análise de normalidade dos dados foi realizada por meio do Shapiro-Wilk. Para as comparações entre as variáveis dicotômicas (Setor: Administrativo ou Operacional; Horas extras: cumpre ou não horas extras) utilizou-se o Teste "t" de Student para amostras independentes. Para a variável carga horária semanal dividida em três categorias (Até 40 horas, de 41 a 55 horas e, 56 ou mais) realizou-se uma análise de variância e o teste de post hoc de Tukey para as comparações múltiplas, se necessário. 


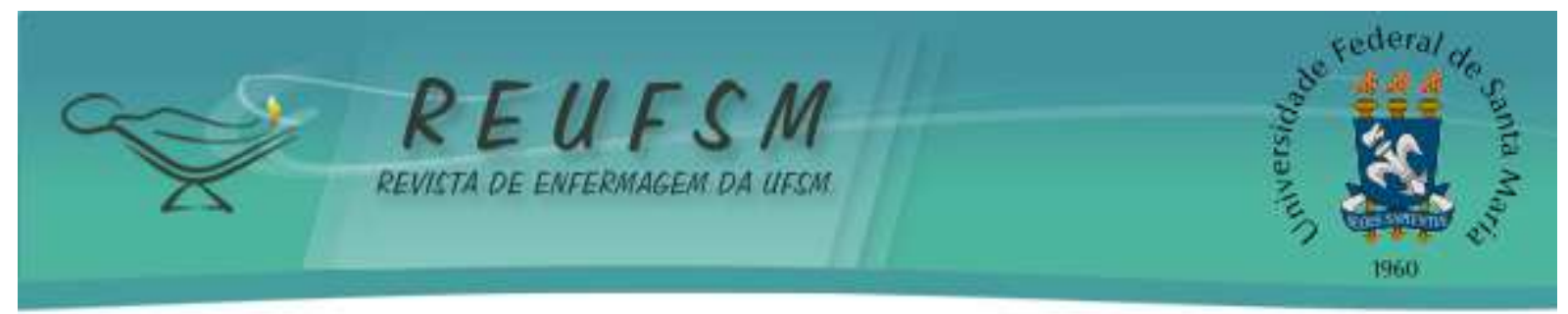

Este estudo seguiu a Resolução 466/2012 do Conselho Nacional de Saúde, que determina as diretrizes e normas regulamentadoras de pesquisa envolvendo seres humanos, ${ }^{7} \mathrm{e}$ foi aprovado pelo Comitê de Ética em Pesquisa da Universidade do Estado de Santa Catarina - UDESC em 17 de fevereiro de 2016, sob CAAE: 51375815.0.0000.0118. Foi garantido o anonimato aos participantes, esclarecido objetivo e metodologia do estudo, com posterior assinatura do termo de consentimento livre e esclarecido.

\section{RESULTADOS}

A população deste estudo foi constituída por 51 participantes. Faz-se necessário esclarecer que o único bombeiro militar vinculado ao $6^{\circ} \mathrm{BBM}-\mathrm{SC}$ do sexo feminino se encontrava em licença maternidade no período da coleta de dados.

Constatou-se que os participantes são em sua maioria casados ou com companheiro $(88,2 \%, \mathrm{n}=42)$, com filhos $(52,9 \%, \mathrm{n}=27)$ e não tabagistas $(94,1 \%, \mathrm{n}=48)$, não apresentaram problemas de saúde no último ano $(72,5 \%, \mathrm{n}=37)$ e não fazem uso de medicamentos $(84,3 \%$, $\mathrm{n}=43)$. Os profissionais, de maneira geral avaliaram que possuem tempo de descanso $(92,2 \%$, $\mathrm{n}=47)$, e avaliaram o tempo para o lazer como suficiente $(68,6 \%, \mathrm{n}=35)$. Embora sinalizaram ausência de período de descanso $(7,8 \%, \mathrm{n}=4)$, o que nos remete a relacionar ao número expressivo de profissionais que realizam horas extras $(67,4 \%, n=33)$.

Quanto aos problemas de saúde informados pelos bombeiros militares estão problemas músculo esquelético $(7,8 \%)$, gastrite $(5,9 \%)$, hérnia de disco $(3,92 \%)$, estresse $(3,9 \%)$, espondilite anquilosante, hepatite, dor cervical, hipertensão, insônia, amigdalite e infecção auditiva com $1,9 \%$ cada.

O maior número de militares no $6^{\circ}$ batalhão foi de soldados, seguido por sargento, cabo, subtenente e tenente $(28 ; 19,6 ; 15,7 ; 5,9$ e $3,9 \%$ respectivamente), lotados em atividades operacionais $(72,5 \%, n=37)$ e administrativas $(27,5 \%, n=14)$. O predomínio da carga horária semanal foi de 56 horas. A jornada de trabalho que prevaleceu foi de 24 horas/dia com descanso de 48 horas, fator que, automaticamente, obriga o bombeiro militar a trabalhar para além das 40 horas semanais.

Quanto à organização do local de trabalho, quase a metade dos participantes julgou parcialmente organizado $(41,2 \%, \mathrm{n}=21)$, com ritmo de trabalho moderado $(64,7 \%, \mathrm{n}=32)$ e número de pessoas na escala de trabalho insuficiente $(82,4 \%, \mathrm{n}=42)$, fato que pode ter 


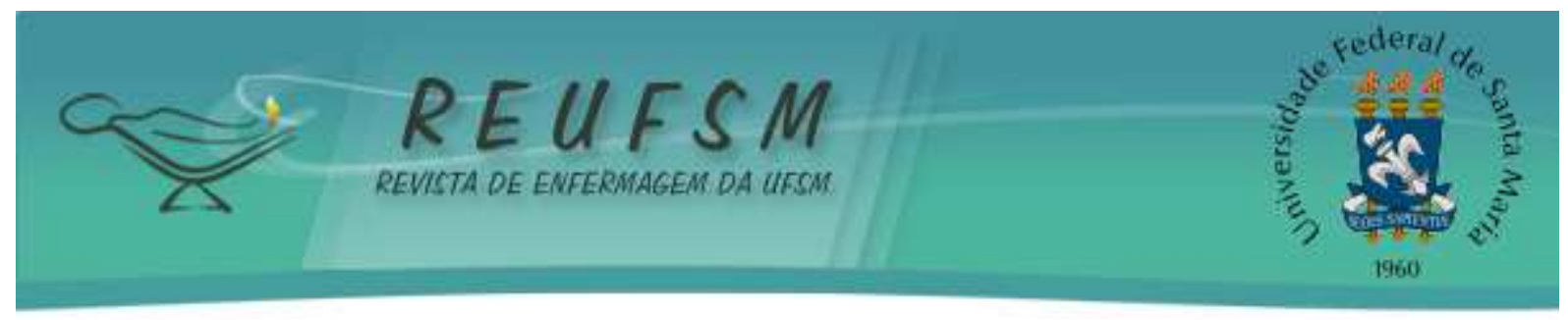

contribuído para a ausência de treinamentos e capacitações no último ano $(45,1 \%, \mathrm{n}=23)$. Além disso, no período de trabalho as horas de sono dos participantes variaram de três a nove horas.

As Tabelas 1, 2 e 3 apresentam os resultados acerca da qualidade de vida dos BM e satisfação com a saúde (SS). Nesse sentido, faz-se necessário esclarecer que o $\mathrm{N}$ não é igual em todas as variáveis analisadas porque há itens que não foram respondidos pelos participantes deste estudo.

A tabela 1 apresenta a percepção dos bombeiros sobre a qualidade de vida e satisfação com sua vida em função do setor de trabalho.

Tabela 1 - Avaliação da percepção da qualidade de vida, satisfação com sua vida em função do setor de trabalho. SC, Brasil, 2016

\begin{tabular}{lllllllll}
\hline \multicolumn{1}{c}{ Domínios } & \multicolumn{4}{c}{ Administrativo(N=14) } & \multicolumn{4}{c}{ Operacional(N=37) } \\
& $\mathrm{N}$ & $\%$ & Média & DP & N & $\%$ & Média & DP \\
\hline PGQV & 14 & 27,4 & $3,86^{*}$ & 0,66 & 36 & 70,6 & 3,39 & 0,18 \\
SV & 13 & 25,4 & 3,85 & 0,90 & 37 & 72,5 & 3,86 & 0,67 \\
Domínio Físico & 14 & 27,4 & 3,99 & 0,38 & 34 & 66,7 & 3,74 & 0,58 \\
Domínio Psicológico & 14 & 27,4 & 4,15 & 0,46 & 35 & 68,6 & 3,88 & 0,44 \\
Domínio das Relações Sociais & 14 & 27,4 & 3,98 & 0,59 & 37 & 72,5 & 3,73 & 0,63 \\
Domínio do Ambiente & 14 & 27,4 & $3,87^{*}$ & 0,52 & 33 & 64,7 & 3,54 & 0,45 \\
\hline
\end{tabular}

Fonte: Banco de Dados dos Autores (2016)

$* \mathrm{p}<0,05$ - Teste " $\mathrm{t}$ " de Student

DP - Desvio Padrão

PGQV - Percepção geral da qualidade de vida

SV - Satisfação com sua vida

A percepção geral da qualidade de vida foi significativamente maior $(\mathrm{p}<0,05)$ entre os bombeiros que atuam no setor administrativo do que os que atuam no setor operacional. Também foi significativamente maior $(\mathrm{p}<0,05)$ entre os que não fazem horas extras do que entre aqueles que fazem (Tabela 2). Por outro lado, a satisfação com a saúde não foi diferente $(p>0,05)$ entre os setores operacional e administrativo e entre os bombeiros que fazem e os que não fazem horas extras.

As medidas de tendência central relativas aos escores de qualidade de vida indicam que tanto os trabalhadores do setor administrativo quanto operacional, percebem sua qualidade de vida geral como "Regular". No entanto, os maiores valores foram alcançados no domínio psicológico, sendo este domínio considerado "Bom" para os trabalhadores do setor administrativo. 


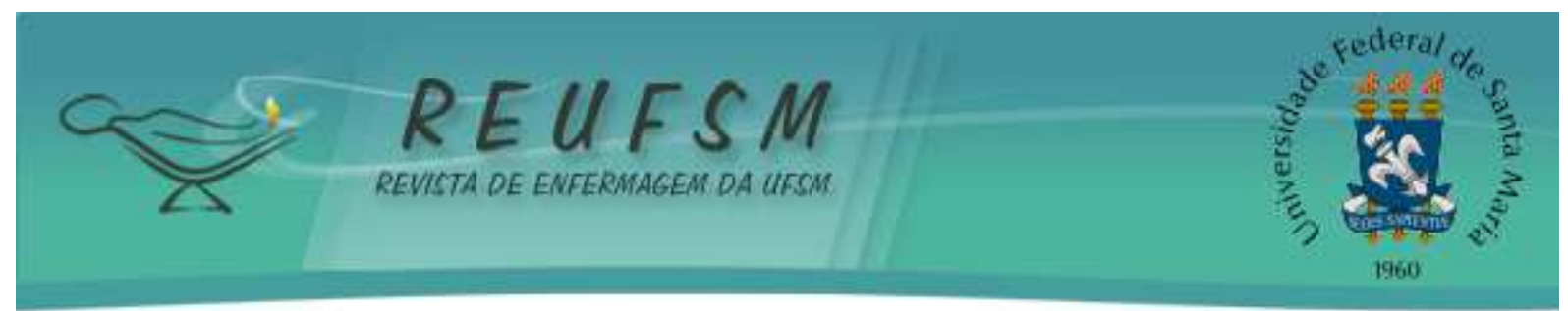

Em relação ao domínio do ambiente, os trabalhadores do setor administrativo apresentaram valores significativamente maiores que o setor operacional $(\mathrm{p}<0,05)$. Tal domínio apresentou os menores escores para os dois locais de trabalho investigados (Tabela 1). Contudo, os trabalhadores do administrativo apresentaram índices maiores que os trabalhadores do operacional em todos os domínios.

A tabela 2 apresenta a percepção dos bombeiros sobre a qualidade de vida e satisfação com sua vida em função da realização de horas extras.

Tabela 2 - Avaliação da percepção da qualidade de vida e da satisfação com sua vida em função das horas extras. SC, Brasil, 2016

\begin{tabular}{lcccccccc}
\hline \multicolumn{1}{c}{ Domínios } & \multicolumn{4}{c}{ Não faz horas extras } & \multicolumn{4}{c}{ Faz horas extras } \\
& $\mathrm{N}$ & $\%$ & Média & $\mathrm{DP}$ & $\mathrm{N}$ & $\%$ & Média & DP \\
\hline PGQV & 26 & 51 & 3,73 & 0,67 & 24 & 47 & 3,29 & 0,69 \\
SV & 26 & 51 & 3,96 & 0,72 & 24 & 47 & 3,75 & 0,74 \\
Domínio Físico & 27 & 52,9 & $3,99 *$ & 0,44 & 21 & 41,2 & 3,59 & 0,57 \\
Domínio Psicológico & 26 & 51 & $4,13^{*}$ & 0,36 & 23 & 45 & 3,76 & 0,49 \\
Domínio das Relações & 27 & & $4,04^{*}$ & 0,52 & 24 & 47 & 3,53 & 0,64 \\
Sociais & 26 & 51 & $3,80^{*}$ & 0,47 & 21 & 41,2 & 3,44 & 0,44 \\
Domínio do Ambiente & 26 & & & & & &
\end{tabular}

Fonte: Banco de Dados dos Autores (2016)

* Diferença estatisticamente significante $(\mathrm{p}<0,05)$

DP - Desvio Padrão

PGQV - Percepção geral da qualidade de vida

SV - Satisfação com sua vida

Ao analisar a percepção da qualidade de vida em função da realização ou não de horas extras, os bombeiros que não fazem horas extras apresentaram diferença estatisticamente significativa $(\mathrm{p}<0,05)$ sobre os que realizam horas extras nos domínios Físico, Psicológico, Relações Sociais e Meio Ambiente (Tabela 2), sendo que os domínios Psicológicos e Relações Sociais foram avaliados como "Boa", enquanto os domínios Físico e Meio Ambiente como "Regular". Já os trabalhadores que fazem horas extras avaliaram sua qualidade de vida como "Regular" em todos os domínios do WHOQOL-Bref.

Na tabela 3 apresenta-se a percepção dos bombeiros sobre a qualidade de vida e satisfação com sua vida decorrente da carga horária semanal de trabalho. 


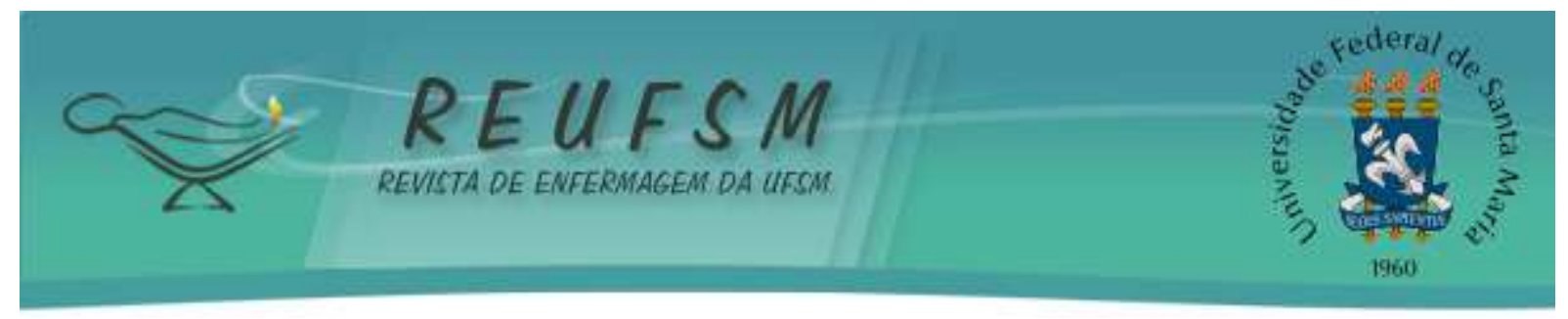

Tabela 3 - Avaliação da percepção da qualidade de vida e da satisfação com sua vida em função da carga horária semanal de trabalho. SC, Brasil, 2016

\begin{tabular}{lcccccccccccc}
\hline Domínios & \multicolumn{4}{c}{ Até 40 horas } & \multicolumn{4}{c}{ De 41 a 55 horas } & \multicolumn{5}{c}{56 ou mais horas } \\
& N & $\%$ & Média & DP & N & $\%$ & Média & DP & N & $\%$ & Média & DP \\
\hline PGQV & 15 & 29,4 & 3,67 & 0,82 & 14 & 27,4 & 3,36 & 0,63 & 18 & 35,3 & 3,56 & 0,62 \\
SV & 14 & 27,4 & 3,79 & 0,80 & 14 & 27,4 & 3,86 & 0,53 & 19 & 37,2 & 4,00 & 0,67 \\
Físico & 15 & 29,4 & 3,96 & 0,46 & 13 & 25,5 & 3,87 & 0,43 & 17 & 33,3 & 3,71 & 0,61 \\
Psicológico & 14 & 27,4 & 4,11 & 0,43 & 13 & 25,5 & 3,94 & 0,41 & 19 & 37,2 & 3,96 & 0,40 \\
Relações & 15 & 29,4 & 4,02 & 0,61 & 14 & 27,4 & 3,79 & 0,36 & 19 & 37,2 & 3,74 & 0,60 \\
Sociais & & & & & & & & & & & & \\
Ambiente & 14 & 27,4 & 3,79 & 0,52 & 14 & 27,4 & 3,69 & 0,30 & 16 & 31,3 & 3,58 & 0,41 \\
\hline
\end{tabular}

Fonte: Banco de Dados dos Autores (2016)

* Análise de Variância

DP - Desvio Padrão

PGQV - Percepção geral da qualidade de vida

SV - Satisfação com sua vida

A análise da percepção geral da qualidade de vida e da satisfação com a saúde em função da carga horária semanal de trabalho não evidenciou nenhuma diferença estatisticamente significante ( $\mathrm{p}>0,05)$ entre os três grupos analisados (Tabela 3).

Considerando a jornada de trabalho semanal de até 40 horas, os Domínios Psicológicos e Relações Sociais foram avaliados como Boa qualidade de vida, enquanto que de 41 a 55 horas semanais e 56 ou mais horas de trabalho semanal, todos os domínios foram percebidos como "regular" (Tabela 3). Contudo, não foram encontradas diferenças estatisticamente significativas para a variável carga horária semanal em nenhum dos domínios analisados.

\section{DISCUSSÃO}

Ao considerar que diferentes fatores podem contribuir para o sofrimento psíquico com repercussão na qualidade de vida do trabalhador, tais como as demandas da organização do trabalho: ritmo, jornada, monotonia ou repetitividade, situações de emergência, autonomia, capacidade de respostas para as adversidades laborais, recursos e tecnologias, contribuindo para o aumento do sofrimento no trabalho e aparecimento de distúrbios físicos e emocionais, ${ }^{8}$ o presente estudo evidencia alguns problemas de saúde, tais como, gastrite, estresse, insônia, entre outros, os quais podem decorrer das atividades laborais e consequentemente influenciar a percepção geral de qualidade de vida. 


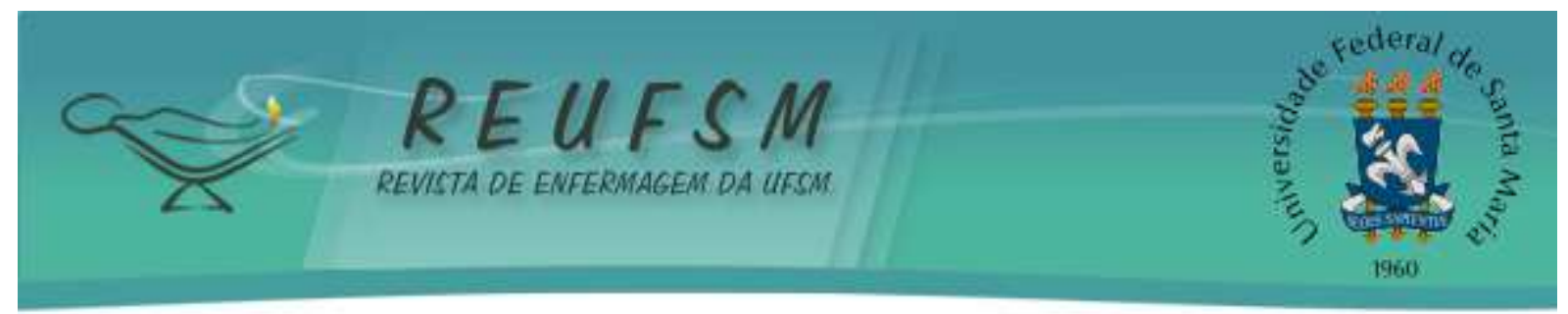

Jornadas de trabalho prolongadas podem levar a falta de tempo para descanso, lazer e até mesmo para o trabalho doméstico, bem como comprometer a qualidade de vida, ${ }^{9}$ corroborando com os achados deste estudo. Ainda, o excesso de trabalho, baixa remuneração e más condições laborais são apontadas como fatores que favorecem o adoecimento psíquico, ${ }^{9}$ o que desperta para os resultados deste estudo acerca da ausência de período para descanso, jornada de trabalho de 24 horas/dia repercutindo na quantidade de profissionais que realizam horas extras, provavelmente pelo número insuficiente de profissionais na escala de trabalho, além dos achados quanto a organização e ritmo de trabalho.

As consequências das longas jornadas incluem o tempo insuficiente para o repouso e para o lazer. Houve um aumento da intensidade do trabalho em si nos últimos anos, e como consequência, o aumento da desorganização do tempo de não trabalho com prejuízos à saúde do trabalhador. ${ }^{10}$

As doenças originadas pelo trabalho são normalmente identificadas em estágios mais avançados por apresentarem sinais e sintomas comuns a outras doenças, ${ }^{8}$ o que nos remete a refletir acerca da insônia e na quantidade de horas de sono sinalizadas pelos participantes deste estudo e o desenvolvimento de estresse. $\mathrm{O}$ trabalho pode levar a desequilíbrios físicos e mentais, favorecendo o adoecimento, ${ }^{8}$ resultando em maiores riscos para ocorrer acidentes de trabalho.

Os resultados apontam para fatores que podem levar ao adoecimento, tais como a carga horária, ritmo de trabalho e quantidade de horas de sono. Estudo sinaliza que o trabalho pode causar agravos à sua saúde quando jornadas vão além de 48-50 horas por semana, causando riscos na segurança do trabalhador, podendo gerar acidentes. ${ }^{11} \mathrm{E}$, indiretamente, o excesso de trabalho pode apresentar desdobramentos negativos no convívio fora da atividade laboral ${ }^{10}$ e também na própria produtividade, uma vez que pode favorecer a ocorrência de problemas no atendimento aos indivíduos. ${ }^{12}$

Percebe-se que alguns participantes do estudo têm um período de sono inferior às necessidades fisiológicas e, considerando que este possui a função de restaurar e conservar a energia do indivíduo, a sua privação traz prejuízos às atividades do dia a dia. ${ }^{13}$

As recomendações das horas de repouso para os trabalhadores são de, no mínimo seis (6) horas diárias, contudo algumas pessoas precisam dormir mais do que isso, além de seguir algumas práticas para um bom descanso, ${ }^{14}$ pois o repouso é fundamental no processo de recuperação do desgaste físico e mental do indivíduo após o período de vigília. Assim, faz-se 


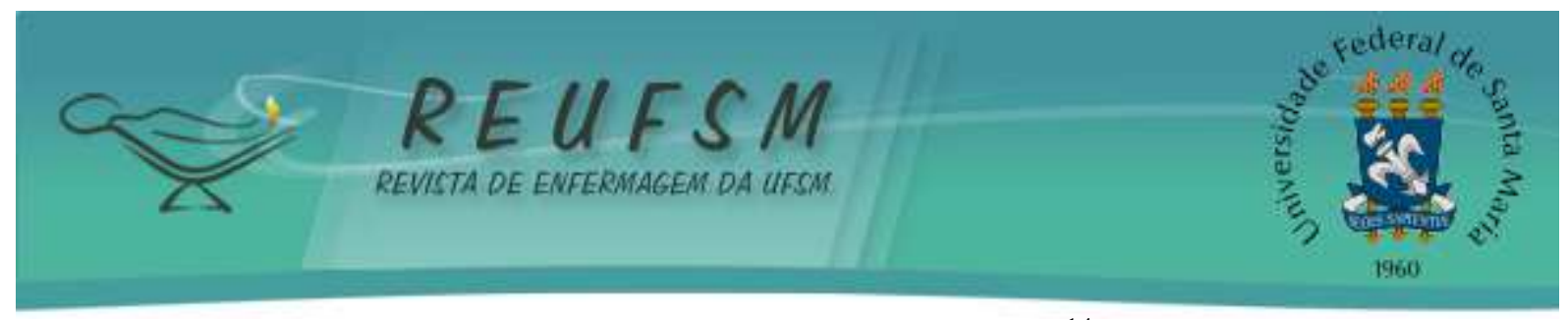

necessário certo equilíbrio, evitando a privação de sono, ${ }^{14}$ uma vez que esta pode comprometer o desempenho cognitivo e memória, além de resultar em alteração de humor e período de sonolência diurna involuntária. ${ }^{15}$

Nesse sentido, a redução prolongada de sono leva ao surgimento de efeitos adversos à saúde, tais como o aumento do risco de depressão, diabetes, obesidade e doenças cardiovasculares, 15 sendo o trabalho por turnos, horário de trabalho irregular e o estresse fatores importantes que induzem a má qualidade do sono ou sua diminuição. ${ }^{15}$ Nesse sentido, os profisssionais que não fazem horas extras apresentaram melhor percepção da qualidade de vida e satisfação com sua vida quando comparado aos que realizam horas extras. Da mesma forma, quem realiza até 40 horas semanais percebem a QV como "Boa", enquanto os que realizam mais de 40 horas semanais percebem sua QV como "Regular" em todos os domínios do WHOQOL-Bref.

Durante as atividades laborais o bombeiro pode sugerir frieza diante de acontecimentos, para que não se faça transparecer as fraquezas e sofrimento psicológico, uma vez que tais situações exigem do profissional boas condições física e psicológica para o bom atendimento a sociedade. Nesse sentido, estudo realizado com militares no Rio de Janeiro sinalizou a associação entre sofrimento psíquico e alguns fatores, tais como a capacidade de reagir a situações difíceis e grau de satisfação com a vida; problemas de saúde e; condições adversas de trabalho, como carga excessiva, constante exposição ao estresse e à vitimização. ${ }^{16}$

Estudo que analisou a presença de sofrimento psíquico e o nível de qualidade de vida por meio do WHOQOL-Bref, entre Policiais Militares de Operações Especiais do município de Patos-PB, sugere cautelosamente que, quanto maior a exigência e sobrecarga houver nas demandas laborais, mais fortemente os sintomas somáticos se apresentam, repercutindo em maior sofrimento físico e mental. ${ }^{8}$

Diversos fatores podem afetar a qualidade de vida, entre eles, ter dois empregos, carga horária elevada, trabalhar sob pressão, alimentação inadequada, sedentarismo e dormir pouco, além das atividades desgastantes inerentes a própria profissão, ${ }^{17}$ sendo que neste estudo, alguns participantes informaram ter dois empregos, realizar horas extras e dormir pouco, fatores que contribuem para o envelhecimento precoce. ${ }^{17}$

A organização temporal do trabalho nos batalhões faz com que os bombeiros sejam expostos a horários atípicos como já citados: turnos de 24 horas, seguidos de 48 horas de folga, somando aproximadamente 56 horas de trabalho semanais. Considerando a natureza 


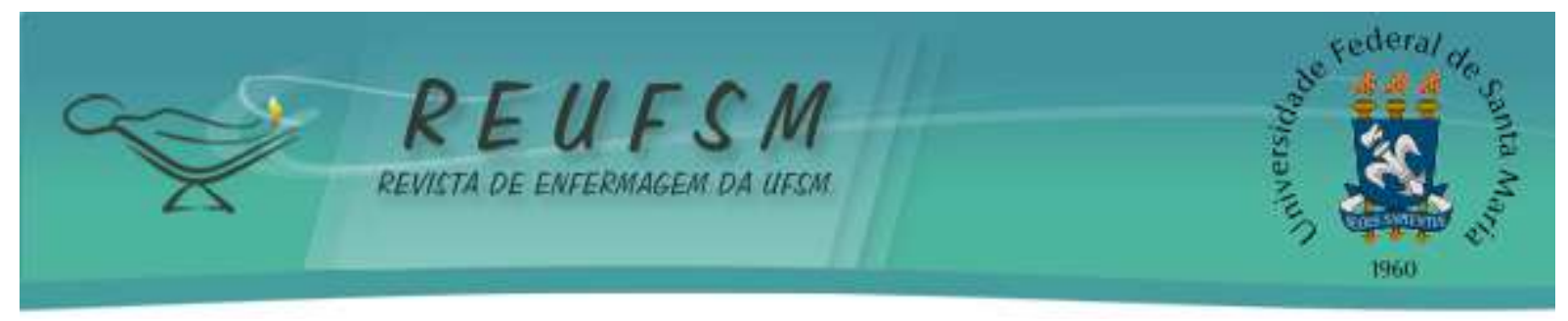

das tarefas e os resultados obtidos neste estudo, tal cenário nos remete a um contexto de alta demanda e trabalho sobre pressão temporal. ${ }^{18}$

Pesquisa realizada com 38 bombeiros para identificar o nível de qualidade de vida do bombeiro militar que atua na região do entorno sul do Distrito Federal constatou que 79\% avaliaram sua qualidade de vida como boa e muito boa, ${ }^{19}$ divergindo dos achados deste estudo, em que a PGQV foi avaliada como "Regular" em todos os domínios pelos bombeiros do setor operacional.

Estudo que investigou a percepção da qualidade de vida de 316 militares masculinos, que exerciam a atividade na área operacional na região metropolitana de Belo Horizonte, apresentaram os maiores escores de qualidade de vida nos domínios Relações Sociais e Psicológico, quando comparados aos domínios físico e meio ambiente, ${ }^{20}$ enquanto neste estudo os melhores escores foram evidenciados nos domínios Físico e Psicológico.

Características da organização temporal do trabalho, com longas jornadas de trabalho trazem efeitos diretos na redução do tempo disponível para outras atividades, aumentando a exposição às longas demandas e favorecendo os riscos no trabalho. ${ }^{10}$

As jornadas extensas de trabalho trazem dificuldades nas relações sociais, pessoais e ainda influenciam na falta de tempo para o lazer e o cuidado com a saúde, podendo ser associada a comportamentos inadequados de saúde como aumento no consumo de álcool, tabagismo, redução de exercício físico e padrão inadequado de sono/distúrbios do sono. ${ }^{21} \mathrm{~A}$ sobrecarga de trabalho tem sido uma das variáveis mais apontadas como preditora para o desenvolvimento de agravos a saúde do trabalhador. ${ }^{22}$

Estudo $^{22}$ que investigou a qualidade de vida de docentes universitários também constatou níveis de qualidade de vida Regular e sinaliza que o resultado encontrado pode ser reflexo da sobrecarga física e psicológica imposta pelas responsabilidades do trabalho, possivelmente fruto do ambiente e das condições de trabalho, resultado que corrobora com os achados desta investigação.

Sugere-se intervenções pontuais, sobretudo no domínio meio ambiente e na questão da realização de horas extras, visando manter o bem-estar desses trabalhadores para que se mantenham ativos e contribuindo com a comunidade local, para garantir o cumprimento da missão constitucional de preservar vidas e bens alheios. Ainda, considera-se uma limitação do 


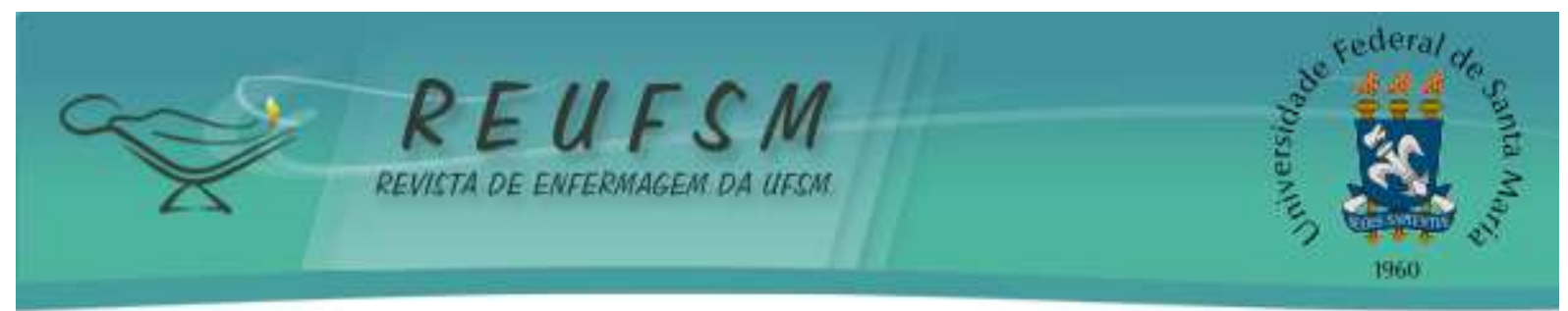

estudo a dificuldade na argumentação baseada em literatura internacional em decorrência da não localização de estudos com esta população.

As limitações do estudo estão relacionadas ao tamanho da população, decorrente do número de efetivos no quadro funcional do cenário investigado.

\section{CONCLUSÃO}

Embora se conheça o difícil processo que envolve a mensuração da qualidade de vida, o presente estudo possibilitou a análise descritiva desta, no âmbito militar. Os resultados obtidos permitem inferir que, de forma geral, os bombeiros militares apresentaram níveis Regulares de qualidade de vida, embora o domínio psicológico tenha revelado diferença significativa entre os setores de trabalho administrativo e operacional.

No domínio Meio Ambiente foram encontrados os piores valores, sendo que os participantes avaliaram sua qualidade de vida como regular em todos os domínios do WHOQOL-Bref.

Os achados deste estudo comprovam equivalência com outras pesquisas, os quais carecem de investimentos em saúde do trabalhador. Neste estudo, a realização de horas extras interferiu negativamente na qualidade de vida do bombeiro militar, resultado pelo qual, sugere-se investigação mais aprofundada para ampliar as discussões sobre qualidade de vida.

\section{REFERÊNCIAS}

1. Miguel V, Varas N, Queirós C. Satisfação com o trabalho como preditor do Burnout em bombeiros assalariados. Porto: Portugal, Instituto de Sociologia da Universidade do Porto. Intl J Working Conditions (RICOT); 2014 [acesso em 2016 ago 04];8:98-113. Disponível em: http://doczz.com.br/doc/238957/satisfa\%C3\%A7\%C3\%A3o-com-o-trabalho-como-preditordo-burnout-em.

2. Santa Catarina. Constituição do Estado. Edição atualizada com 49 Emendas Constitucionais. Florianópolis: Assembleia Legislativa; 2009. 253 p.

3. The Whoqol Group. The World Health Organization Quality of Life assessment (WHOQOL): position paper from the world health organization. Social Sci Med [Internet]. 1995 [acesso em 2016 ago 06];41(10):1403-9. Disponível em: https://www.ncbi.nlm.nih.gov/pubmed/8560308.

4. Silva IEG, Silva BCS, Jesus BP, Reis LHF, Pestana PRM, Silva TEA, et al. Qualidade de vida entre bombeiros militares: estudo epidemiológico. Rev Digital [Internet]. 2013 [acesso em 2018 jan 24];18(182). Disponível em: http://www.efdeportes.com/efd182/qualidade-devida-entre-bombeiros-militares.htm. 


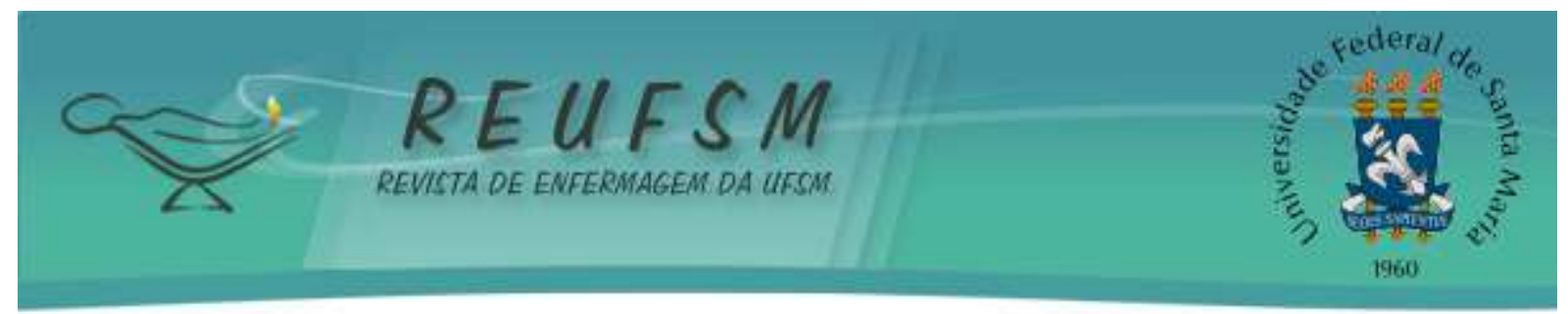

5. Brasil. Ministério da Saúde. Organização Pan-Americana da Saúde no Brasil. Doenças relacionadas ao trabalho: manual de procedimentos para os serviços de saúde. Brasília: Editora MS; 2001 [acesso em 2016 ago 02]. (Série A. Normas e Manuais Técnicos; 114). Disponível

em:

http://bvsms.saude.gov.br/bvs/publicacoes/doencas_relacionadas_trabalho1.pdf.

6. Fleck MPA. O instrumento de avaliação de qualidade de vida da Organização Mundial da Saúde (WHOQOL-100): características e perspectivas. Ciênc Saúde Colet [Internet]. 2000 [acesso em 2016 abr 08];5(1):33-8. Disponível em: http://www.scielo.br/pdf/csc/v5n1/7077.pdf.

7. Brasil. Ministério da Saúde. Conselho Nacional de Saúde. Resolução CNS no 466, de 12 de dezembro de 2012 [acesso em 2016 abr 09]. Aprovar as seguintes diretrizes e normas regulamentadoras de pesquisas envolvendo seres humanos. Disponível em: http://bvsms.saude.gov.br/bvs/saudelegis/cns/2013/res0466_12_12_2012.html.

8. Lucena AKA, Gois OP, Pessoa CMCC, Sousa MNA. Sofrimento psíquico e qualidade de vida entre policiais militares do batalhão de operações especiais. Rev Interdisciplinar Saúde [Internet]. 2015 [acesso em 2018 jan 24];2(1):157-76. Disponível em: http://interdisciplinaremsaude.com.br/Volume_3/Trabalho_10.pdf.

9. Hoelz L, Campello L. Relação entre Síndrome de Burnout, erro médico e longa jornada de trabalho em residentes de medicina. Rev Bras Med Trab [Internet]. 2015 [acesso em 2018 mar 18];13(2):126-34. Disponível em: http://files.bvs.br/upload/S/16794435/2015/v13n2/a5281.pdf.

10. Cardoso ACM. Organização e intensificação do tempo de trabalho. Rev Soc Estado [Internet]. 2013 [acesso em 2018 jan 24];28(2):351-74. Disponível em: http://www.scielo.br/pdf/se/v28n2/v28n2a09.pdf.

11. Lee S, McCann D, Messenger JC. Organização Internacional do Trabalho (OIT). Duração do trabalho em todo o mundo: tendências de jornadas de trabalho, legislação e políticas numa perspectiva global comparada. Brasília: OIT- Secretaria Internacional de Trabalho; 2009 [acesso em 2016 abr 11]. 252 p. Disponível em http://www.ilo.org/brasilia/publicacoes/WCMS_229714/lang--pt/index.htm.

12. Dalri RCMB, Silva LA, Mendes AMOC, Robazzi MLCC. Carga horária de trabalho dos enfermeiros e sua relação com as reações fisiológicas do estresse. Rev Latinoam Enferm [Internet]. 2014 [acesso em 2018 jan 25];22(6):959-65. Disponível em: http://www.scielo.br/pdf/rlae/v22n6/pt_0104-1169-rlae-22-06-00959.pdf.

13. Bacelar A, Pinto Júnior LR, colaboradores. Insônia: do diagnóstico ao tratamento - III Consenso da Associação Brasileira do Sono. Associação Brasileira do Sono (Absono); 2013.

14. Neves GSM, Giorelli AS, Florido P, Gomes MM. Transtornos do sono: visão geral. Rev Bras Neurol [Internet]. 2013 [acesso em 2018 jan 24];49(2):57-71. Disponível em: http://files.bvs.br/upload/S/0101-8469/2013/v49n2/a3749.pdf.

15. Porkka-Heiskanen T, Zitting K-M, Wigren H-M. Sleep, its regulation and possible mechanisms of sleep disturbances. Acta Physiol [Internet]. 2013 [acesso em 2018 mar 18];208(4):311-28. Disponível em: https://www.ncbi.nlm.nih.gov/pubmed/23746394. 


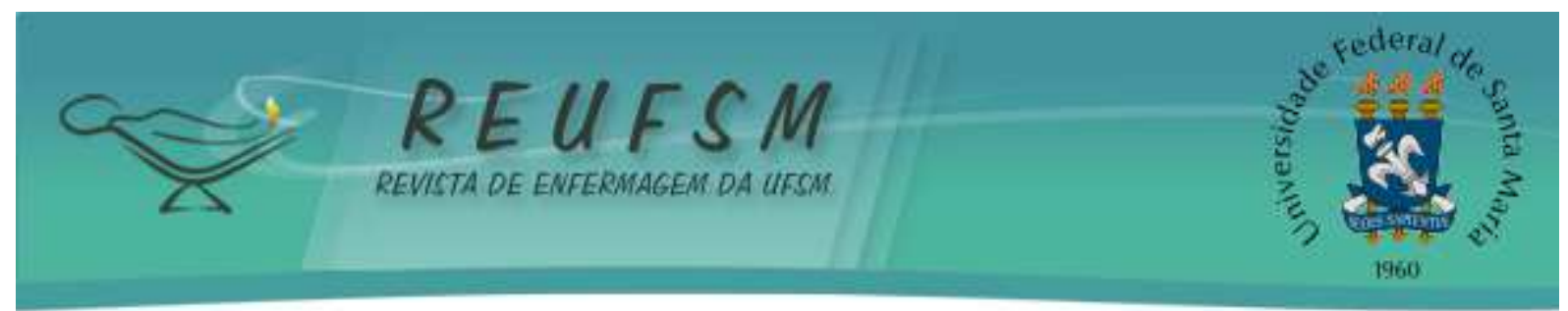

16. Souza ER, Minayo MCS, Silva JG, Pires TO. Fatores associados ao sofrimento psíquico de policiais militares da cidade do Rio de Janeiro, Brasil. Cad Saúde Pública [Internet]. 2012 [acesso em 2018 jan 25];28(7):1297-311. Disponível em: http://www.scielo.br/pdf/csp/v28n7/08.pdf.

17. Minayo MCS, Assis SG, Oliveira RVC. Impacto das atividades profissionais na saúde física e mental dos policiais civis e militares do Rio de Janeiro (RJ, Brasil). Ciênc Saúde Colet [Internet]. 2011 [acesso em 2016 out 12];16(4):2199-209. Disponível em: http://www.scielo.br/pdf/csc/v16n4/v16n4a19.

18. Lima EP, Assunção AÁ, Barreto SM. Transtornos de estresse pós-traumáticos (TEPT) em bombeiros de Belo Horizonte, Brasil: prevalência e fatores ocupacionais associados. Belo Horizonte: Psicol Teoria Pesqu [Internet]. 2015 [acesso em 2016 abr 12];31(2):279-88. Disponível em: http://www.scielo.br/pdf/ptp/v31n2/0102-3772-ptp-31-02-0279.pdf.

19. Mateus AD. Qualidade de vida profissional do bombeiro militar que atua na região do entorno sul do Distrito Federal. Goiania: Corpo de Bombeiros Militar do Estado de Goiás, Curso Superior de Bombeiro Militar; 2013 [acesso em 2016 abr 20]. Disponível em: https://www.bombeiros.go.gov.br/wp-content/uploads/2016/08/tc_adval.pdf.

20. Souza Filho MJ, Noce F, Andrade AGP, Calixto RM, Albuquerque MR, Costa VT. Avaliação da qualidade de vida de policiais militares. Rev Bras Ciência Mov [Internet]. 2015 [acesso em 2016 maio 04];23(4):159-69. Disponível em: http://dx.doi.org/10.18511/01031716/rbcm.v23n4p159-169.

21. Fernandes JC, Portela LF, Rotenberg L, Griep RH. Jornada de trabalho e comportamentos de saúde entre enfermeiros de hospitais públicos. Rev Latinoam Enferm [Internet]. 2013 [acesso em 2016 abr 12];21(5):[08 telas]. Disponível em: http://www.scielo.br/pdf/rlae/v21n5/pt_0104-1169-rlae-21-05-1104.pdf.

22. Santos MPG, Silva KKD. Níveis de estresse e qualidade de vida de professores do ensino superior. Rev Enferm UFSM [Internet]. 2017 out/dez [acesso em $2017 \mathrm{dez}$ 17];7(4):656-68. Disponível em: https://periodicos.ufsm.br/reufsm/article/view/25906/pdf.

Data de submissão: 22/12/2017

Data de aceite: $12 / 06 / 2018$

Autor correspondente: Rosana Amora Ascari

E-mail: rosana.ascari@hotmail.com ou rosana.ascari@udesc.br

Endereço: Rua 14 de Agosto 807 E, Apartamento 301, Bairro: Presidente Médice.

CEP: 89.801-251, Chapecó/SC, Brasil. 